\title{
Designing Data to be Inclusive: Enabling Cross-Disciplinary and Participative Processes
}

\author{
Alastair S. Macdonald and David Loudon \\ School of Design, The Glasgow School of Art, \\ 167 Renfrew Street, Glasgow G3 6RQ, Scotland, UK \\ \{a.macdonald, d. loudon\} @gsa.ac.uk
}

\begin{abstract}
The data formats of specialist disciplines are often difficult for those from other disciplines to access, not least lay audiences, inhibiting truly participative and inclusive processes. The authors discuss the achievements and value of visualizing biomechanical data on functional demand obtained during daily activities from older adults in the $60+, 70+$ and $80+$ age groups which has shown the potential to provide physiotherapists, occupational therapists, designers, bioengineers, and human factors specialists much improved access to the data. Older adults have their own valuable insights and these are vital to include in developing a fuller understanding of issues that affect their quality of life. The format of visualization has the potential to be understood by this 'lay' audience. The paper discusses the design of, and pilots towards a full-scale study to try to fully engage stakeholder disciplines and older people as truly collaborative partners, together with conclusions to date.
\end{abstract}

Keywords: Visualizing data, participative processes, functional demand, older people, quality of life.

\section{Introduction}

Transformation design and participative design processes have much to offer the cause and advancement of inclusive design (ID) also known as universal design (UD). Transformation design is described as design that facilitates collaboration between many disciplines and ordinary people in order to solve complex problems, placing the user at the heart of new systems development (Winhall, 2006). Participative design is a form of democratic participation in the processes and methods of design.

In the UK, research councils emphasize the need for, and value of, greater interdisciplinary collaboration to address ageing population challenges. However, the formats and domain-specificity of the languages and data of specialist disciplines are such that they can often create a barrier to, and are difficult to access by other disciplines, not least by a lay audience. One of the challenges is to make not only processes participative, but also the information and insights of individual disciplines accessible across other disciplines. This would go some way to empowering all stakeholders, including lay contributors, and may help the shift of perception from an 
individual 'user' viewed as a 'subject' who is 'consulted', to a true 'participant' viewed as a contributing 'expert' in his or her own right.

This paper provides details of a case study of a research project in progress describing the potential value of the visualization of biomechanical data in a format that will allow older adults themselves potentially equal access to the data, along with other relevant disciplines, enabling them to be true collaborators. Older adults have their own valuable insights which complement those of specialist disciplines and which are essential to consider to develop a full understanding of issues that affect their quality of life. This paper discusses the achievements of this visualization method, the design of, and pilots towards, a full-scale study, and insights to date.

\subsection{Problems Associated with Ageing, Problems with Data}

Adults as they grow older may have difficulties in performing normal daily living tasks, due to decreasing mobility, injury, or the chronic diseases associated with ageing. A clear understanding of the functional demand placed on the muscles and joints of older adults as they perform these tasks could provide insights for appropriate design solutions for the home (Coleman, 2001), assistive technologies and exercise (Cress et al 1999; Krebbs et al 1998; Laukkenen et al 1998); rehabilitation; and healthcare strategies to maintain independence and quality of life (Woods et al, 1999). However, data processed by bioengineers and by physiotherapists tends to be in a format that is numerical, conveyed in static graphs, and where the dynamic quality and the context in which the data is generated are not communicated effectively. This limits the ability of different subject disciplines, each with related interests, to share and discuss the information and to develop new insights related to quality of life issues of older people.

\section{The Process of Envisioning Dynamic Data}

The original intention of previous research (Loudon and Macdonald, 2005, Macdonald et al, 2006a) was to develop a CAD tool for designers using data calculated from the biomechanical functional demand on joints in older adults during activities of daily living. The proposition was that the presentation of this data in a format understandable to designers would lead to better designs for older people. Whole body movements of older adults in 60+, 70+, and $80+$ age groups were captured using a 3D motion capture camera system, reaction forces were measured by force platforms, and translated into a 3D software model. The rationale for the selection of daily living tasks, and a description of methods of data capture and visualization are described in detail in Macdonald et al (2006b).

\subsection{Animated Data}

To achieve the usability and accessibility of the data, and to create visualizations which could be presented to designers for subsequent evaluation, a prototype software tool was developed, implemented in Visual C++ and OpenGL, which displayed realtime generated 3D animated visualizations of each of the individual participants each performing the 11 activities of everyday living (described in Section 3). The animated 
human model consisted of simple cylindrical or block representations of the body segments from the motion capture rendered frame by frame in three dimensions in light blue. The hands were displayed as 3D hand models shaded as flesh tone. The tool enables the viewer to select a participant (selection by age and gender) and an everyday living task and view a 3D animated model of the participant performing that task. Reanimating the motion capture data provides the viewer with the opportunity to alter the viewpoint to any angle and zoom in or out to examine the motion.

\subsection{Visualizing Functional Demand}

The researchers developed an innovative means of envisioning this data which lies in the way that functional demand data is presented in a visual manner, and in a dynamic form, based on established principles of information visualization. The level of functional demand - how hard the muscles were working relative to their maximum capability - was mapped to a colour scale, based on the analogy of traffic lights (red for 100\% demand and green for 0\% demand).

The colour-coded functional demand was represented by a variable-colour sphere at each of the joints of an animated digital mannequin of each older person, rendered from motion capture data (Figure 1). A green colour was shown where functional demand was below $40 \%$. At higher functional demand, but still within acceptable limits (between $40 \%$ and $80 \%$ ), the shades of colour of the sphere were in the range of green/yellow to orange. Finally, if the joint was experiencing functional demand levels deemed to be unacceptable (above 80\%), the sphere was shaded red. This 'traffic light' system was thought to be a clear and immediate way of allowing the designer to understand, without specialist knowledge, the functional demands placed on individuals while performing daily activities.

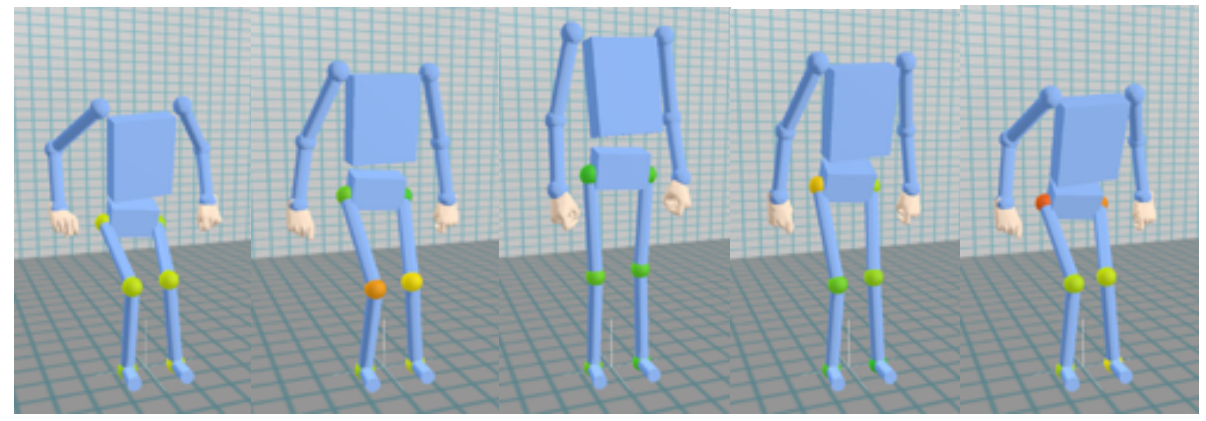

Fig. 1. Software tool displays the functional demand at the joints of the lower limbs during sitting task

The envisioning of the dynamic data allowed one to gain an insight into the relationship between the continuously changing functional demand at each of the joints and the movement of the individual. An understanding of the points at which the greatest functional demand is placed on joints during a movement cycle is easily and quickly identified through this form of visualization. If the viewer requires the 
details of the forces which are being represented, s/he can select the joint to obtain the numerical values, direction of the forces, etc.

\section{Enabling Single- and Cross-Disciplinary Participation}

The potential value of this innovative mode of envisioning dynamic data was identified through working with processed data sets for eight individuals each performing 11 separate daily living tasks, involving e.g. gait, pushing and pulling a door open, sitting/standing, lifting/setting down objects on shelves, ascending/ descending stairs - some tasks with and without the use of arms. The perspectives of those from different backgrounds, i.e., human factors consultants, physiotherapists, and biomechanics were sought in pilot evaluation studies. From these sessions it was found that while each derived new insights from the visualization method, each viewed and interpreted the data with a different emphasis.

\subsection{Pilot 1: Physiotherapists' Perspective}

The pilot for the physiotherapists' evaluation provides a good illustration of the potential value of this form of visualized data: “...that's fascinating actually... one can pick up different methods which they use to circumvent the demand on their hip...the positions they put themselves in... they'll come forward on their ankles more...how they change their centre of gravity...”, “... he's keeping this right knee flexed, because he's probably got OA [osteoarthritis] in that knee...so he's a wee bit rotated ... but there's more force going down through his left ankle. Because he's offloading it...and then he gets to fully standing... and then there's a bit of internal rotation...you can get with hip problems as well. So with him if you were going to design something...let's assume he has OA in his right knee, clinically, and you're...he's not going to be able to put $100 \%$ functional performance through his right knee. He's only able to do 70 before pain inhibits him. So any adaptation isn't going to change anything on the affected side but may improve things on the unaffected side...reduce the loading."

\subsection{Pilot 2: Interdisciplinary Perspective}

An interdisciplinary pilot (bioengineering, physiotherapy and design) has also been undertaken and this again provided useful insights into the common and divergent ways of viewing the data. For instance, in one discussion facilitated by these animations, a bioengineer, who was critical of currently available chairs for older adults, queried a designer about how decisions were made about seat height and arm rests. The bioengineer could specify what would be most suitable for, say, $90 \%$ of older people to be able to push themselves out of a chair. The physiotherapist noticed a new aspect: the hands and feet of an individual were static during one of the movements and if the hands had done more or if the feet were inclined then it would have improved their performance. The bioengineer commented that if they had problems, say in their leg, then they might adapt their movement, introducing coping strategies in order to avoid soreness. 


\subsection{Insights from Pilots}

From these initial pilots, data envisioned in this format appears to be useful to more professions than just the designers for whom it was originally intended. It appears that this format can allow those separate disciplines involved in designing for and the rehabilitation, healthcare and well-being of older people to view the data in an innovative, useful, and sharable manner. This potentially facilitates new insights informed by inter-disciplinary analysis and discussion of the visualized data. These insights have the potential, in turn, to inform any and all of the disciplines, e.g. the bioengineer (in understanding the biomechanical issues), the designer of the built environment (for the design of furniture, homes, care institutions), or the physiotherapist (deciding exercise regimes to improve muscle strength, exercise or rehabilitation equipment). This could provide a comprehensive and holistic view of individuals' needs.

Further reaction was gained from two public feedback events, one involving all the stakeholders including older people, some of whom had been the subjects of the data collection experiments. The informal response from the latter event highlighted the fact that the non-professional lay audience (i.e., the older people themselves) was enabled to make a contribution through this innovative format of data design and presentation.

\section{The Design of Further Studies}

The data for the 84 individuals, each performing 11 set tasks has been collected and is of good quality; the method for processing data has been proven, the software scripts have been written, and the software for processing data is in place. However, as the pilots involved data visualized from only eight individuals, the idiosyncrasies in these data sets do not allow useful generic insights to be gained and meaningful conclusions to be drawn.

The next stage will involve processing and envisioning the remaining data, and using this material (potentially some 924 animations, from which a subset will be chosen) as the basis for a series of participative workshops involving the stakeholder disciplines, and older people, both individually (discipline-specific) and collectively (cross-disciplinary).

\subsection{Engaging Further Perspectives Through Participative Workshops}

The study is being designed as a series of workshops structured using participative methods and which include older participants throughout the whole process. The first set will be single-discipline specific (i.e. human factors consultants, bioengineers, physiotherapists, occupational therapists, designers, etc). At each of these workshops a group of older participants will attend. The thorough briefing of all participants and the sequencing of events will be important. Ascertaining which activities of daily living amongst the older adult participants cause most problems would be crossreferenced to the those activities selected for the original data-acquisition as a result of literature review. A clear briefing on the meaning of the colour-coding used in the animations and its correspondence with the level of demand on joints during the daily 
living tasks will be essential, as will be any implications associated with high (i.e. amber to red) levels of demand on any of the joints. Extrapolating the implications of certain activities along with the diminution of muscle strength with age from, say, the $60+$ cohort to the $70+$ and $80+$ cohorts may prove valuable in predicting safe practice and better-designed environments. The psychological and aspirational implications on quality of life and wellbeing from unsafe or undesirable loading on joints will be evaluated to inform recommendations for safe and meaningful work activities and exercise routines for older adults.

The second set of workshops will be cross-disciplinary in nature, again with older participants. This first phase is described as the co-research phase, to gain insights and appreciate the different perspectives.

During a further phase of the project, the co-design phase, the hope is that sufficient feedback and insights are obtained, a) at a practical level, to generate healthcare and design recommendations and possibly guidelines, b) at a policy level, to develop healthcare and design strategies, and c) at a strategic level, to develop guidelines and recommendations for the acquisition, processing and envisioning of biomechanical data.

The study being described in this section is currently being developed and results will be reported at a later date.

\section{Conclusions}

This project is being undertaken with the aspiration of designing data in such a way as to be 'inclusive', to facilitate improved participation by a range of different specialists, designers, and a lay audience (in this case the older adult), in what were previously discipline-exclusive discussions largely excluding the subjects of their research. The intriguing aspect of this research is to discover to what extent the researchers will be able to liberate normally discipline-specific data and present this in a format to elevate those individuals and groups previously regarded as 'subjects' to be more equal participants and partners - and 'experts through experience' in their own right - in the more inclusive and democratic design of those factors influencing wellbeing and quality of life, here specifically with regard to physical activities of daily living for older adults.

Acknowledgments. The initial phase of this research was supported by the Engineering and Physical Sciences Research Council Grant GR/R26856/01, made to the Bioengineering Unit and the Department of Psychology at the University of Strathclyde in Glasgow, the School of Health Sciences at Queen Margaret University College in Edinburgh, and Product Design Engineering at The Glasgow School of Art.

\section{References}

1. Coleman, R.: Living longer: the new context for design. Design Council, London (2001)

2. Cress, M.E., Buchner, D.M., Questad, K.A., Esselman, P.C., DeLateur, B.J., Schwartz, R.S.: Exercise: effects on physical functional performance in independent older adults. Journals of Gerontology: Biological Sciences and Medical Sciences 54(5), 242-248 (1999) 
3. Krebs, D.E., Jette, I.M., Assmann, S.F.: Moderate exercise improves gait stability in disabled elders. Arch Phys. Med Rehab. 79, 1489-1495 (1998)

4. Laukkanen, P., Kauppinen, M., Heikkinen, E.: Physical activity as a predictor of health and disability in 75 and 80 year old men and women. Journal of Aging and Physical Activity 6(2), 141-156 (1998)

5. Loudon, D., Macdonald, A.S.: Software tool for designers. Include 2005, Helen Hamlyn Research Centre, London (April 2005)

6. Macdonald, A.S., Loudon, D., Rowe, P.J., Samuel, D., Hood, V., Nicol, A.C., Conway, B.A.: InclusiveCAD: a software resource for designers. In: Clarkson, J., Langdon, P., Robinson, P. (eds.) Designing accessible technology, London, pp. 93-99. Springer, Heidelberg (2006)

7. Macdonald, A.S., Loudon, D., Rowe, P.J., Samuel, D., Hood, V., Nicol, A.C., Grealy, M.A., Conway, B.A.: Towards a design tool for visualizing the functional demand placed on older adults by everyday living tasks. In: Universal Access in the Information Society (in press), Springer, Berlin (2006)

8. Winhall, J.: Is design political? Cited accessed 30/01/07. (2006) Available at http://www.core77.com/reactor/03.06_winhall.asp

9. Wood, R.H., Reyes-Alvarez, R., Maraj, B., Metoyer, K., Welsh, M.: Physical fitness, cognitive function and health-related quality of life in older adults. Journal of Aging and Physical Activity 7(3), 217-230 (1999) 